\title{
Fatores associados para potenciais interações medicamentosas clinicamente significantes em terapia intensiva adulto
}

\author{
Associated factors for potential clinically significant drug-drug interactions in \\ adult intensive care
}

Juliano Teixeira Moraes ${ }^{1}$ (D), Juliana Moreira Maia² (D), Olívia Maria Trindade² (D), Luiz Alberto de Oliveira ${ }^{3}$ (D), Cristina Sanches ${ }^{4}$ (D), Danilo Donizetti Trevisan ${ }^{5}$ (D)

\begin{abstract}
RESUMO
Modelo do estudo: Estudo transversal. Objetivo: caracterizar as interações medicamentosas potenciais (IMp) maiores e contraindicadas em terapia intensiva adulto e determinar sua prevalência, medicamentos e fatores associados à exposição dessas interações. Métodos: a amostra foi composta por 309 pacientes internados em uma unidade de terapia intensiva adulto de um hospital da Região Centro-Oeste de Minas Gerais, Brasil. Os dados demográficos-clínicos e perfil medicamentoso foram coletados, prospectivamente. As IMp foram caracterizadas pela base Micromedex 2.0 quanto à gravidade, prevalência e implicação clínica. Realizaram-se análises descritivas para as variáveis qualitativas e contínuas. Modelos Lineares Generalizados com distribuição Gama e Poisson foram utilizados para avaliar a relação entre um conjunto de variáveis independentes e a prevalência de IMp maiores e contraindicadas $(p<0.05)$. Resultados: Um total de $81,8 \%(n=251)$ foi exposto a, no mínimo, uma IMp maior ou contraindicada. Mais de um terço (37,4\%) destes participantes foram expostos a seis ou mais IMp. Medicamentos com ação no sistema nervoso colaboraram para maior probabilidade de IMp maiores e/ou contraindicadas. Pessoas idosas $(p=0,006)$, do sexo masculino $(p=0,028)$ e polimedicadas $(<0,001)$ tiveram maior probabilidade de serem expostas ao menos a uma IMp maior ou contraindicada. Conclusão: Interações medicamentosas potenciais maiores e contraindicadas apresentaram alta frequência. Pessoas idosas, do sexo masculino e polimedicadas foram mais expostas às IMp maiores e/ou contraindicadas. É importante que profissionais da saúde conheçam os riscos e potenciais eventos adversos relacionados às IMp maiores e contraindicadas, para que sejam implementadas medidas para promoção da segurança do paciente.
\end{abstract}

Palavras-chave: Interações de Medicamentos; Eventos Adversos; Unidade de Terapia Intensiva; Cuidados Críticos; Segurança do Paciente.

\begin{abstract}
Study Design: Cross-sectional. Objective: characterizing major and contraindicated potential drug-drug interactions (PDDI) in adult intensive care as well as determining their prevalence, risk factors, and potentially high-risk medications. Methods: The sample was composed of 309 patients hospitalized in an adult intensive care unit in the Midwest Region of Minas Gerais state, Brazil. Clinical data and drug profile were prospectively collected. The PDDI were characterized using the Micromedex 2.0 as to severity, prevalence, and clinical implications. Descriptive analyses were performed for qualitative and continuous variables. Generalized Linear Models with Gamma and Poisson distribution were used to assess the relationship between a set of independent variables and the prevalence of major and contraindicated PDDI $(p<0.05)$. Results: A total of $81.8 \%(n=251)$ were exposed to, at least, a major or contraindicated PDDI. More than a third (37.4\%) of these participants were exposed to six or more IMp. Drugs with action on the nervous system contributed to a greater probability of major and/or contraindicated PDDI. Elderly $(p=0.006)$, male $(p=0.028)$, and polymedicated $(<0.001)$ people were more likely to be exposed to at least a higher or contraindicated PDDI. Conclusion: Potentially major and contraindicated drug interactions showed high frequency. Elderly, male, and polymedicated people were more exposed to larger and/or contraindicated PDDI. It is important that health professionals are aware of the risks and potential adverse events related to major and contraindicated PDDI so that measures might be implemented to promote patient safety.

Keywords: Drug Interactions; Adverse Events; Intensive Care Units; Critical Care; Patient Safety.

Doutor em Ciências Aplicadas à Saúde do Adulto, Professor Adjunto da Universidade Federal de São João del-Rei, Divinópolis (MG), Brasil. Acadêmico em Medicina, Universidade Federal de São João del-Rei, Divinópolis (MG), Brasil.

Enfermeiro, Universidade Federal de São João Del-Rei, Divinópolis (MG), Brasil.

Doutora em Ciências Farmacêuticas, Professora Adjunta da Universidade Federal de São João del-Rei, Divinópolis (MG), Brasil.

Doutor em Enfermagem, Professor Adjunto da Universidade Federal de São João del-Rei, Divinópolis (MG), Brasil.

$\bowtie$ Juliano Teixeira Moraes. Universidade Federal de São João del-Rei / Campus Centro Oeste. Av. Sebastião Gonçalves Coelho, 400 - sala 304.4 Bloco D - Chanadour. CEP: 35501-296. Divinópolis (MG), Brasil.

julianotmoraes@ufsj.edu.br | Recebido em: 07/07/2020 | Aprovado em: 03/08/2020
\end{abstract}




\section{INTRODUÇÃO}

Interações medicamentosas (IMs) são interações que ocorrem entre combinações de medicamentos utilizados para diferentes indicações ou tratamentos e podem resultar em consequências não intencionais com alterações na farmacocinética e/ou farmacodinâmica, acarretando na diminuição da eficácia ou no aumento da toxicidade do medicamento ${ }^{1,2}$. São consideradas eventos adversos evitáveis, podem apresentar desfechos graves e/ou fatais e comprometer a segurança do paciente ${ }^{3,4}$.

As consequências clínicas provenientes das IMs são difíceis de serem identificadas devido ao número de medicamentos administrados de forma concomitante e do quadro clínico do paciente (por exemplo, gravidade da doença e presença de comorbidades). Deste modo, dada a incerteza da ocorrência ou não de uma IM, neste estudo considerou-se a potencialidade deste evento ocorrer e utilizou-se o termo "Interação Medicamentosa potencial" (IMp) ${ }^{5}$.

Pacientes hospitalizados em unidades de terapia intensiva (UTI) são altamente expostos a IMs devido a sua gravidade, presença de comorbidades, agudização de doenças crônicas, alterações em órgãos envolvendo metabolização/excreção de medicamentos e necessidade de incrementar o número de medicamentos prescritos devido ao regime terapêutico ${ }^{1,6}$.

A prevalência de IMp em UTI tem sido elevada, variando de 58 a $96 \%{ }^{6-10}$. Estudos ${ }^{6,11}$ têm reportado que os principais fatores de risco e associados a estas taxas envolvem ser do sexo masculino, idade maior de 60 anos, uso de polifarmácia incluindo algumas classes de medicamentos específicas (antiagregantes plaquetários, antidiabéticos, antiarrítmicos, diuréticos, inibidores de bomba de prótons, hipnóticos/sedativos e antiepilépticos) e maior tempo de internação6,11.

Diversos estudos observacionais ${ }^{7-16}$ caracterizaram as IMp bem como sua frequência e fatores associados em UTI. No entanto, não foram encontradas estimativas consistentes da prevalência de IMp consideradas clinicamente significantes (com potencial de acarretar riscos fatais) por pacientes bem como dos medicamentos, fatores associados e potencias impactos clínicos em pacientes hospitalizados em UTI adulto. Assim, este estudo propõe caracterizar as interações medicamentosas potenciais clinicamente significantes em terapia intensiva adulto e determinar sua prevalência, medicamentos e fatores associados à exposição dessas interações.

\section{MATERIAL E MÉTODOS}

\section{Tipo e local do estudo}

Este é um estudo transversal e seguiu as recomendações da Declaração STROBE ${ }^{17}$. Os dados foram coletados em uma UTI adulto de um hospital filantrópico de grande porte localizado na região CentroOeste do estado de Minas Gerais, Brasil. A instituição é referência para 54 municípios desta região e mais de $70 \%$ de seus atendimentos são realizados pelo Sistema Único de Saúde (SUS). Possui pouco mais de 350 leitos ativos e atende todos os níveis de complexidades dentro das seguintes especialidades: Clínica Médica, Cirurgia, Ginecologia e Obstetrícia, Terapia Intensiva Adulto, Infantil e Neonatal, Nefrologia, Oncologia, e Urgência e Emergência.

A pesquisa obedeceu aos critérios éticos para pesquisas com seres humanos da resolução 466/2012 sendo aprovada pelo Comitê de Ética local por meio dos pareceres $\mathrm{n}^{\circ} 1.096 .400$ e 1.114.269.

\section{Seleção da amostra e coleta de dados}

Os participantes foram recrutados consecutivamente no período de julho a dezembro de 2015. Foram incluídos pacientes com idade igual ou maior de 18 anos, internados em unidade de terapia intensiva adulto, no mínimo há 24 horas, e que faziam uso de pelo menos dois ou mais medicamentos.

Para o cálculo amostral, considerou-se como parâmetro de interesse a prevalência de IMp. Considerando esta variável, as seguintes definições para o cálculo do tamanho da amostra foram utilizadas: (i) nível de confiança de $95 \%$; (ii) prevalência de exposição à IMp de $72,5 \%^{9}$; (iii) erro máximo de $5 \%$. Assim, a amostra calculada foi de 309 participantes.

Os dados coletados, prospectivamente, foram extraídos dos prontuários dos pacientes por um instrumento elaborado pelos autores. A variável dependente foi a IMp, ou seja, a potencialidade de apresentar uma interação medicamentosa, 
proveniente dos medicamentos prescritos. As variáveis independentes foram divididas em dois grupos: demográfico-clínicas (sexo, idade e especialidade médica) e perfil medicamentoso (nome genérico dos medicamentos e polifarmácia). Medicamentos fitoterápicos, à base de vitaminas ou sais minerais e componentes de dieta enteral ou parenteral não foram incluídos no estudo.

\section{Classificação das interações medica- mentosas potenciais}

Os medicamentos foram inseridos na Base de Dados Micromedex @ 2.0 para identificar e analisar as IMp. Este software tem a sensibilidade, especificidade e confiabilidade adequadas para detectar IMp. O Micromedex ® 2.0 fornece os pares de IMp a partir da combinação dos medicamentos e as categoriza de acordo com a gravidade, implicação clínica potencial e manejo clínico ${ }^{18}$. Em relação ao nível de gravidade, foram consideradas IMp clinicamente significantes aquelas categorizadas como contraindicada (o uso concomitante dos medicamentos não é recomendado) ou maior (quando a interação apresenta ameaça à vida e/ou requerer intervenção médica para reduzir ou prevenir eventos adversos sérios). A análise das implicações e manejos clínicos foi realizada pela leitura do sumário da IMp disponibilizado na base de dados por dois pesquisadores; em seguida, cada implicação e manejo foram agrupados por similaridades.

Para categorizar os medicamentos prescritos, foram utilizados os níveis 1 e 5 da AnatomicalTherapeutic-Chemical Classification (ATC) da Organização Mundial da Saúde (OMS) ${ }^{19}$.

\section{Análise dos dados}

Os dados coletados foram transferidos para uma planilha do Microsoft Excel $2010 \AA$, por dupla digitação. Estatísticas descritivas foram utilizadas para resumir as características demográfico-clínicas, perfil medicamentoso e prevalência das interações, respeitando-se a distribuição das variáveis (aplicado teste de Kolmogorov-Smirnov).
Realizaram-se comparações do número de IMp maiores e/ou contraindicada com cada medicamento identificado pelo teste de Mann-Whitney. Em seguida, com os medicamentos relacionados estatisticamente significantes $(p<0,05)$ pelo teste de Mann-Whitney, se ajustou um modelo linear generalizado (MLG) com distribuição Gama e função de ligação canônica inversa para avaliar quais destes medicamentos independentemente mais predizem as IMp maiores e/ou contraindicada.

Por fim, outro MLG com distribuição Poisson com variância robusta e função de ligação logarítmica foi aplicado para avaliar a relação entre um conjunto de variáveis independentes e a exposição às IMp. Foram apresentadas as estimativas obtidas da razão de prevalência, juntamente com seus respectivos intervalos de confiança e p-valor. Não foram identificados outliers. Um nível de significância de $5 \%$ foi utilizado em todos os testes. Os dados foram analisados usando o software Statistical Package for the Social Sciences (SPSS- versão 23.0).

\section{RESULTADOS}

\section{Características demográfico-clínicas e perfil medicamentoso}

Em um período de seis meses, foram incluídos 309 prontuários, sendo 172 (55,7\%) de pacientes do sexo masculino, com mediana de idade de 67 anos (intervalo interquartil $=21,0$ ) e em maioria $(89,0 \%)$ acompanhados por especialidades clínicas.

A mediana de medicamentos prescritos foi de 7,0 (intervalo interquartil $=5,0$ ). Foram identificadas 115 substâncias ativas distintas e totalizando 2348 medicamentos prescritos. A maioria dos medicamentos foi classificada nos grupos A $(n=703-29,9 \%), C(n=632-26,9 \%)$ e $N(n=498-21,2 \%)$ daclassificaçãoATC. Osdezmedicamentos mais frequentemente prescritos por paciente foram metoclopramida $(53,7 \%)$, ranitidina $(48,2 \%)$, enoxaparina $(44,9 \%)$, sinvastatina $(37,5 \%)$, furosemida $(33,1 \%)$, omeprazol $(31,4 \%)$, dipirona $(31,1 \%)$, insulina $(22,6 \%)$, captopril $(21,7 \%)$, ácido acetilsalicílico (21,3\%). Na Tabela 1 são apresentadas as características demográfico-clínicas e perfil medicamentoso encontrados nos prontuários. 


\section{Tabela 1}

Caracterização demográfico-clínica e perfil medicamentoso de pacientes expostos a interações medicamentosas potenciais maiores e contraindicadas $(n=309)$

\begin{tabular}{|c|c|}
\hline Variáveis & n (\%) \\
\hline \multicolumn{2}{|l|}{ Sexo } \\
\hline Masculino & $172(55,7)$ \\
\hline Feminino & $137(44,3)$ \\
\hline \multicolumn{2}{|l|}{ Idade } \\
\hline$<60$ & $102(33,0)$ \\
\hline$\geq 60$ & $207(67,0)$ \\
\hline \multicolumn{2}{|l|}{ Medicamentos } \\
\hline$<5$ & $48(15,5)$ \\
\hline$\geq 5$ & $261(84,5)$ \\
\hline \multicolumn{2}{|l|}{ Especialidade } \\
\hline Cirúrgica & $34(11,0)$ \\
\hline Clínica & $275(89,0)$ \\
\hline \multicolumn{2}{|l|}{ Classificação ATC } \\
\hline $\begin{array}{l}\text { A - ação no aparelho digestivo e } \\
\text { metabolismo }\end{array}$ & $703(29,9)$ \\
\hline $\begin{array}{l}\text { B- agentes que envolvem sangue e } \\
\text { órgãos hematopoiéticos }\end{array}$ & $154(6,5)$ \\
\hline C - ação no sistema cardiovascular & $632(26,9)$ \\
\hline H - preparações hormonais sistêmicas & $46(1,8)$ \\
\hline J - agentes anti-infecciosos & $167(7,1)$ \\
\hline N - ação no sistema nervoso & $498(21,2)$ \\
\hline R - ação no aparelho respiratório & $148(6,3)$ \\
\hline
\end{tabular}

\section{Interações medicamentosas potenciais maiores e contraindicadas}

A prevalência de pacientes expostos a pelo menos uma IMp maior ou contraindicada foi de $81,8 \%$ $(n=251)$; entre estes pacientes, $64,1 \% \quad(n=161)$ eram pessoas idosas e $35,9 \%(n=90)$ adultos. Quase a metade dos participantes $(41,4 \%)$ foi exposta de uma a três interações, $24,6 \%$ de quatro a seis interações e $15,2 \%$ a sete ou mais interações (mediana de 3,0; intervalo interquartil $=4,0$ ). Um total de 1076 pares de IMp foram identificadas, sendo que $94,9 \%$ $(\mathrm{n}=1021)$ foram classificadas com gravidade maior $\mathrm{e}$ $5,1 \%(n=55)$ contraindicada.

Por meio de comparações pelo teste de Mann-Whitney entre o número de IMp maiores ou contraindicadas com cada medicamento prescrito $(n=115)$, pode-se observar diferença estatisticamente significante $(p<0,05)$ de medianas de IMp maiores ou contraindicadas entre pacientes que utilizaram 20 medicamentos. Em seguida, se ajustou um modelo linear generalizado (MLG) com distribuição Gama e função de ligação inversa, indicando como variáveis explicativas os medicamentos previamente significativos estatisticamente e variável desfecho o número de IMp maiores e/ou contraindicadas. Conclui-se que a administração de ondansetrona, clopidogrel, amiodarona, captopril, losartana, metilprednisolona, dipirona, fenobarbital, fentanil, midazolam, morfina e tramadol foram os medicamentos mais propensos para expor os pacientes às IMp maiores e contraindicadas mais elevadas (Tabela 2).

As estimativas que constam na Tabela 2 devem ser interpretadas como variação no inverso da média (intercepto) da variável desfecho. Ou seja, a média de IMp por pacientes que não utilizaram nenhum desses 20 medicamentos foi $1 /(0,523)=1,91$. Se o paciente utilizou ondansetrona, por exemplo, o inverso da média reduz 0,096, isto é: $1 /(0,523-0,096)=2,35$. Deste valor subtrai-se a constante de 1,91 obtendo-se um aumento de 0,44. Deste modo, pacientes que utilizaram ondansetrona foram expostos a uma média de IMp maior e/ou contraindicada 0,44 vezes mais elevada comparados com aqueles que não utilizaram este medicamento.

Medicamentos com ação no sistema nervoso associados à metoclopramida foram os que acarretaram uma maior frequência de pacientes expostos às IMp contraindicadas e maiores, como apresentadas na Tabela 3. As implicações clínicas potenciais mais frequentes estiveram relacionadas com aumento dos riscos de reações extrapiramidais e de depressão do sistema nervoso central, de sangramento, de nefrotoxicidade e de arritmias.

No MLG com distribuição Poisson e ligação logarítmica, pode-se evidenciar que a probabilidade de participantes idosos e do sexo masculino serem expostos ao menos a uma IMp maior e/ou contraindicada foi 1,14 e 1,12 vezes, respectivamente, a probabilidade dos participantes idosos $(p=0,006)$ e do sexo feminino $(p=0,028)$ serem expostos. Utilizar menos de cinco medicamentos foi um fator de proteção para pacientes internados em UTI não serem expostos ao menos a uma IMp maior e/ou ou contraindicada $(R P=0,55$; $\mathrm{p}<0,001$ ) (Tabela 4). 


\section{Tabela 2}

Modelo linear generalizado com distribuição Gama e ligação inversa entre o número de interações medicamentosas maiores ou contraindicadas e uso de medicamentos $(n=1076)$

\begin{tabular}{|c|c|c|c|c|c|c|}
\hline ATC & Medicamentos & $\beta$ & L.I & L.S & p-valor & Média de aumento de IMp \\
\hline & Intercepto & 0,523 & 0,458 & 0,588 & $<0,001$ & - \\
\hline \multirow{4}{*}{ A } & Insulina & $-0,331$ & $-0,072$ & 0,007 & 0,107 & - \\
\hline & Metoclopramida & $-0,370$ & $-0,082$ & 0,007 & 0,101 & - \\
\hline & Ondansetrona & $-0,096$ & $-0,155$ & $-0,037$ & 0,002 & 0,446 \\
\hline & Ranitidina & $-0,012$ & $-0,048$ & 0,025 & 0,527 & - \\
\hline \multirow{3}{*}{ B } & Ácido acetilsalicílico & $-0,042$ & $-0,097$ & 0,013 & 0,131 & - \\
\hline & Clopidogrel & $-0,091$ & $-0,156$ & $-0,026$ & 0,006 & 0,419 \\
\hline & Enoxaparina & $-0,028$ & $-0,062$ & 0,007 & 0,115 & - \\
\hline \multirow{5}{*}{ C } & Amiodarona & $-0,110$ & $-0,155$ & $-0,066$ & $<0,001$ & 0,527 \\
\hline & Anlodipina & 0,032 & $-0,024$ & 0,088 & 0,258 & - \\
\hline & Captopril & $-0,083$ & $-0,136$ & $-0,031$ & 0,002 & 0,377 \\
\hline & Losartana & $-0,107$ & $-0,202$ & $-0,013$ & 0,026 & 0,509 \\
\hline & Sinvastatina & $-0,020$ & $-0,059$ & 0,019 & 0,309 & - \\
\hline $\mathrm{H}$ & Metilprednisolona & $-0,099$ & $-0,172$ & $-0,026$ & 0,008 & 0,463 \\
\hline \multirow{7}{*}{$\mathrm{N}$} & Dipirona & $-0,093$ & $-0,131$ & $-0,055$ & $<0,001$ & 0,429 \\
\hline & Fenobarbital & $-0,137$ & $-0,184$ & $-0,090$ & $<0,001$ & 0,698 \\
\hline & Fentanil & $-0,134$ & $-0,185$ & $-0,084$ & $<0,001$ & 0,681 \\
\hline & Haloperidol & $-0,029$ & $-0,076$ & 0,018 & 0,231 & - \\
\hline & Midazolam & $-0,095$ & $-0,155$ & $-0,035$ & $<0,001$ & 0,441 \\
\hline & Morfina & $-0,101$ & $-0,153$ & $-0,050$ & $<0,001$ & 0,476 \\
\hline & Tramadol & $-0,111$ & $-0,156$ & $-0,067$ & $<0,001$ & 0,535 \\
\hline
\end{tabular}

ATC: Anatomical Therapeutic Chemical; Modelo Linear Generalizado (MLG) com distribuição Gama

\section{Tabela 3}

Interações medicamentosas potenciais maiores e contraindicadas mais prevalentes em pacientes hospitalizados em unidade de terapia intensiva adulto $(n=309)$

\begin{tabular}{|c|c|c|c|}
\hline \multicolumn{2}{|c|}{ Pares de IMp contraindicadas } & \multirow{2}{*}{ n (\%) } & \multirow{2}{*}{ Implicação clínica potencial } \\
\hline Medicamento A & Medicamento B & & \\
\hline \multirow{7}{*}{ Metoclopramida } & Haloperidol & $18(5,8)$ & \multirow{7}{*}{$\begin{array}{c}\uparrow \text { risco de reações extrapiramidais e, em casos extremos, de } \\
\text { síndrome neuroléptica maligna }\end{array}$} \\
\hline & Clorpromazina & $5(1,6)$ & \\
\hline & Duloxetina & $4(1,3)$ & \\
\hline & Quetiapina & $4(1,3)$ & \\
\hline & Citalopram & $2(0,6)$ & \\
\hline & Amitriptilina & $2(0,6)$ & \\
\hline & Sulpirida & $2(0,6)$ & \\
\hline \multicolumn{2}{|c|}{ Pares de IMp maiores } & \multirow{2}{*}{ n (\%) } & \multirow{2}{*}{ Implicação clínica potencial } \\
\hline Medicamento A & Medicamento B & & \\
\hline \multirow{6}{*}{ Metoclopramida } & Tramadol & $39(12,6)$ & \multirow{6}{*}{$\begin{array}{c}\uparrow \text { risco de reações extrapiramidais e, em casos extremos, de } \\
\text { síndrome neuroléptica maligna }\end{array}$} \\
\hline & Fentanil & $36(11,7)$ & \\
\hline & Fenobarbital & $22(7,1)$ & \\
\hline & Midazolam & $14(4,5)$ & \\
\hline & Diazepam & $8(2,6)$ & \\
\hline & Clonazepam & $6(1,9)$ & \\
\hline \multirow{4}{*}{ Dipirona } & Enoxaparina & $63(20,4)$ & \multirow{6}{*}{$\uparrow$ risco de sangramento } \\
\hline & Ácido acetilsalicílico & $22(7,1)$ & \\
\hline & Varfarina & $6(2,6)$ & \\
\hline & Clopidogrel & $4(1,9)$ & \\
\hline \multirow{2}{*}{ Clopidogrel } & Ácido acetilsalicílico & $18(5,8)$ & \\
\hline & Enoxaparina & $10(3,2)$ & \\
\hline \multirow{2}{*}{ Furosemida } & Dipirona & $46(14,9)$ & \multirow{4}{*}{$\begin{array}{l}\downarrow \text { eficácia diurética e } \\
\uparrow \text { risco de nefrotoxicidade }\end{array}$} \\
\hline & Ácido acetilsalicílico & $28(9,1)$ & \\
\hline \multirow{2}{*}{ Espironolactona } & Dipirona & $13(4,2)$ & \\
\hline & Ácido acetilsalicílico & $12(3,9)$ & \\
\hline \multirow{2}{*}{ Amiodarona } & Sinvastatina & $19(6,1)$ & \multirow{2}{*}{$\uparrow$ risco de alargamento do intervalo QT e arritmias } \\
\hline & Ranitidina & $17(5,5)$ & \\
\hline
\end{tabular}

(Continua...) 
Tabela 3 (Continuação)

\begin{tabular}{|c|c|c|c|}
\hline \multicolumn{2}{|c|}{ Pares de IMp maiores } & \multirow{2}{*}{ n (\%) } & \multirow{2}{*}{ Implicação clínica potencial } \\
\hline Medicamento A & Medicamento B & & \\
\hline \multirow{3}{*}{ Ondansetrona } & Haloperidol & $2(0,6)$ & \multirow{5}{*}{$\uparrow$ risco de arritmias } \\
\hline & Amitriptilina & $2(0,6)$ & \\
\hline & Fluoxetina & $2(0,6)$ & \\
\hline \multirow{2}{*}{ Espironolactona } & Cloreto de potássio & $18(5,8)$ & \\
\hline & Captopril & $12(3,9)$ & \\
\hline \multirow{3}{*}{ Fentanil } & Ranitidina & $30(9,7)$ & \multirow{3}{*}{ †risco de depressão do sistema nervoso central } \\
\hline & Midazolam & $16(5,2)$ & \\
\hline & Haloperidol & $8(2,6)$ & \\
\hline \multirow{2}{*}{ Ranitidina } & Tramadol & $42(13,6)$ & \multirow{2}{*}{$\uparrow$ risco de depressão respiratória } \\
\hline & Metadona & $2(0,6)$ & \\
\hline \multirow{3}{*}{ Sinvastatina } & Anlodipina & $17(5,5)$ & \multirow{3}{*}{$\uparrow$ risco de rabdomiólise e miopatia } \\
\hline & Ciprofloxacina & $4(1,3)$ & \\
\hline & Azitromicina & $3(1,0)$ & \\
\hline
\end{tabular}

IMp: Interação Medicamentosa potencial; Resultados expressos como $\mathrm{n}(\%)$.

\section{Tabela 4}

Modelo linear generalizado considerando distribuição Poisson com variância robusta entre interação medicamentosa potencial e variáveis independentes $(n=309)$

\begin{tabular}{|c|c|c|c|c|c|c|}
\hline \multirow{3}{*}{ Variáveis } & \multicolumn{4}{|c|}{ IMp } & \multirow{3}{*}{ Razão de Prevalência } & \multirow{3}{*}{ p-valor } \\
\hline & \multicolumn{2}{|c|}{ Não } & \multicolumn{2}{|c|}{ Sim } & & \\
\hline & $\mathbf{n}$ & $\%$ & $\mathbf{n}$ & $\%$ & & \\
\hline Idade & & & & & & 0,006 \\
\hline$<60$ anos & 12 & $(3,9)$ & 90 & $(29,1)$ & 1,00 (ref) & \\
\hline$\geq 60$ anos & 46 & $(14,9)$ & 161 & $(52,1)$ & $1,14(1,03-1,25)$ & \\
\hline Sexo & & & & & & 0,028 \\
\hline Feminino & 29 & 9,4 & 108 & $(46,6)$ & 1,00 (ref) & \\
\hline Masculino & 28 & 9,0 & 144 & $(35,0)$ & $1,12(1,02-1,23)$ & \\
\hline Especialidade & & & & & & 0,663 \\
\hline Clínica & 53 & $(17,2)$ & 222 & $(71,8)$ & 1,00 (ref) & \\
\hline Cirúrgica & 5 & $(1,6)$ & 29 & $(9,4)$ & $1,02(0,91-1,16)$ & \\
\hline Número de medicamentos & & & & & & $<0,001$ \\
\hline$\geq 5$ & 34 & $(11,0)$ & 227 & $(73,4)$ & 1,00 (ref) & \\
\hline$<5$ & 24 & $(7,8)$ & 24 & $(7,8)$ & $0,55(0,42-0,73)$ & \\
\hline
\end{tabular}

IMp: Interação Medicamentosa potencial; Modelo Linear Generalizado (MLG) com distribuição Poisson

\section{DISCUSSÃO}

A prevalência de IMp maiores ou contraindicadas identificada neste estudo foi elevada, e os medicamentos mais associados com este desfecho agiam no sistema nervoso e envolviam o sangue e órgãos hematopoiéticos (Grupo N e B da classificação ATC, respectivamente). Uso de polifarmácia, ser idoso e do sexo masculino foram fatores associados com as IMp maiores e/ou contraindicadas em UTI adulto.

Medicamentos que predizem IMp não necessariamente podem acarretar eventos adversos aos pacientes, todavia, o risco para estes danos se eleva quando as IMp são classificadas como clinicamente significantes (maiores e/ou contraindicadas) ${ }^{20}$. Este estudo demonstrou alta prevalência $(81,8 \%)$ de pacientes expostos a pelo menos uma IMp maior ou contraindicada, sendo que mais de um terço desses pacientes $(39,8 \%)$ foram expostos a quatro ou mais destas IMp simultaneamente.

Comparando a estudos prévios que investigaram a ocorrência de IMp em UTI, a prevalência encontrada neste estudo foi similar a de estudos prévios que variou de 58 a $96 \%{ }^{6-10}$. O perfil medicamentoso relacionado às IMp deste estudo corrobora com achados de recente revisão sistemática com metanálise ${ }^{6}$ e de outros estudos observacionais ${ }^{11-16}$ que objetivaram avaliar IMp em UTI. Adicionalmente, este estudo 
identificou e quantificou, por meio de um MLG, quais medicamentos foram os mais preditores para a ocorrência de IMp maiores e/ou contraindicadas. Na UTI analisada, pacientes que utilizaram alguns medicamentos das classes de antieméticos, antiagregantes plaquetários, antiarrítmicos, anti-hipertensivos, analgésicos, anticonvulsivantes, sedativos e opioides, por exemplo, foram expostos a um número mais elevado a estas IMp comparando a pacientes que não os utilizaram (Tabela 2). Destaca-se que o fenobarbital e o fentanil foram os medicamentos que mais estiveram envolvidos para incrementar o número de IMp graves.

Pacientes internados em UTI adulto geralmente são graves e podem necessitar de suporte ventilatório e de sedação; além disso, podem apresentar quadros dolorosos e, deste modo, a administração de sedativos, hipnóticos, analgésico e/ou opioides é fundamental para proporcionar conforto ao paciente, reduzir o estresse e evitar retardo na recuperação ${ }^{21-23}$. Por outro lado, estes medicamentos são classificados como potencialmente perigosos por sua alta probabilidade de acarretar eventos adversos e dano ao paciente $7,24,25$. Desta forma, a implantação de protocolos de uso e prevenção de erros de medicação, aliados a prevenção e acompanhamento de reações adversas são de extrema importância para garantir um ambiente seguro para os pacientes criticamente doentes ${ }^{25,26}$.

Com relação ao uso da dipirona, proibida em muitos países, a segurança da sua indicação continua sendo um tema controverso ${ }^{27}$. Sabe-se que a agranulocitose, mesmo com incidência rara, é seu evento adverso mais preocupante; entretanto, mesmo assim ainda apresenta vantagens com relação a segurança quando comparada a outros analgésicos não esteroidais ${ }^{28}$. Seu uso intra-hospitalar concomitante com ranitidina, heparina e metoclopramida (por exemplo) pode ser considerado prática frequente em hospitais brasileiros ${ }^{12,14,29}$, e, portanto, compõe uma lista dos principais medicamentos prescritos causadores de IMp e contribui com a ocorrência de polifarmácia, como observado neste estudo. Entretanto, tanto a dipirona quanto a metoclopramida por serem utilizadas de forma esporádica e eventual, ou seja, apenas em casos de febre/dor ou vômito (se necessário), não se pode precisar uma relação de causalidade de IMp com estes medicamentos.
O uso de metoclopramida (um agonista dopaminérgico), além das IMp descritas neste estudo, apresenta como principal evento adverso a ocorrência de eventos extrapiramidais quando administrados em altas concentrações ou concomitantemente com agentes antipsicóticos ${ }^{30}$ bem como relatos de agitação, cefaleia e dores abdominais. Adicionalmente, achados de uma revisão sistemática compararam o uso de metoclopramida e placebo com o objetivo de prevenir vômito em pacientes com dor aguda que receberam morfina intravenosa. Os resultados demonstraram baixas evidências de benefícios clínicos do uso deste agonista dopaminérgico e maior probabilidade de acarretar danos ao paciente ${ }^{31}$. Desta forma, a associação de metoclopramida com opioides pode potencializar eventos adversos e contribuir para maior exposição a IMp.

Um estudo retrospectivo descreveu que metade dos eventos adversos relacionados ao uso de medicamentos necessitaram de um maior nível de cuidado, sendo que $74 \%$ destes poderiam proporcionar danos temporários ou permanentes e $84 \%$ eram evitáveis; as principais classes de medicamentos envolvidas foram os antibióticos e agentes antitrombóticos ${ }^{32}$. No presente estudo, a ocorrência de IMp entre os medicamentos do grupo $B$, dentre eles antiagregantes plaquetários e anticoagulantes, apresentando como potencial consequência o risco de sangramento, também foi elevada.

No Brasil estes medicamentos compõem uma lista de medicamentos potencialmente perigosos. Uma ampla discussão tem sido realizada sobre uma avaliação ultraconservadora para tromboprofilaxia, gerando maior risco de danos aos pacientes. Desta forma, assim como para os demais medicamentos apresentados anteriormente, discussões fundamentadas em evidência científica devem balizar a relação necessidade/risco da utilização de medicamentos em pacientes críticos ${ }^{7,24}$.

Deste modo, o manejo das IMp na prática clínica não é tarefa simples. O impacto das IMp poderá sofrer influência de fatores de risco individuais não modificáveis como comorbidades, idade avançada e polimorfismo genético. Por outro lado, estes aspectos podem ser manejados de acordo com a experiência clínica e do nível de reconhecimento da equipe de saúde sobre os riscos das IMp, principalmente as de gravidade maior e contraindicadas. Lamentavelmente, clínicos frequentemente ignoram 
ou subestimam estas interações severas disponíveis nos alertas eletrônicos ${ }^{33}$.

Assim, o manejo das IMp deve incluir o envolvimento de diferentes profissionais de saúde, como enfermeiros, farmacêuticos clínicos e médicos, o uso de prescrições eletrônicas com um sistema de alerta e bancos de dados para identificação de IMp, que podem permitir o rastreamento em tempo real de IM. Destaca-se ser importante considerar o risco-benefício das combinações de medicamentos. O uso de sistemas de alerta precoce para categorização de IMp de acordo com a gravidade em alguns cenários de assistência médica poderá incrementar a aceitação de recomendações clínicas para que certos medicamentos não sejam prescritos simultaneamente ${ }^{1,34}$.

Deste modo, para a prática clínica é fundamental que a equipe multiprofissional aprimore o conhecimento sobre o significado clínico das IMp provenientes de medicamentos utilizados em UTI, tanto para tratar a causa da internação bem como o surgimento de complicações decorrentes do tratamento e comorbidades prévias. Compete à equipe de saúde realizar o monitoramento do paciente, avaliar sinais e sintomas que podem surgir das IMp e conhecer sobre os benefícios e riscos da terapêutica medicamentosa, visando prestar assistência segura ao paciente.

Com pontos fortes, para nosso conhecimento, este estudo foi o primeiro a investigar a prevalência de IMp clinicamente significante em pacientes hospitalizados em UTI adulto, bem como identificar quais medicamentos colaboraram para elevar o número destas IMp e apresentar as principais implicações clínicas potencias que podem ser desencadeadas. Por outro lado, algumas limitações devem ser consideradas. Primeiramente, apenas os medicamentos prescritos para uso na UTI foram registrados; não foram realizadas avaliações clínicas para detecção da ocorrência da IMp ou entrevistas com pacientes e familiares para identificar a prática de automedicação ou tratamentos alternativos. As IMp foram analisadas em uma única base de dados. Outra limitação identificada foi a não classificação da gravidade do paciente bem como o levantamento de comorbidades, exames laboratoriais e de diagnóstico.

\section{CONCLUSÃO}

Constatou-se uma elevada prevalência de IMp clinicamente significantes em prescrições de pacientes internados na UTI adulto avaliada. Os medicamentos mais envolvidos nas IMp foram com ação no sistema nervoso. Pacientes idosos, do sexo masculino e em uso de polifarmácia tiveram maior probabilidade de serem expostos a IMp. Diversas implicações clínicas potenciais foram identificadas, com destaque para o aumento dos riscos de reações extrapiramidais, depressão do sistema nervoso central e sangramento. A identificação e o monitoramento de IMp clinicamente significantes envolvendo todo o sistema de medicação são relevantes para reduzir a ocorrência de evento adversos relacionados a medicamentos e promover a segurança do paciente.

\section{DECLARAÇÃO DE CONFLITOS DE INTERESSE}

Os autores declaram ausência de conflitos de interesse.

\section{FONTE FINANCIADORA DO PROJETO}

Programa Institucional de Bolsas de Iniciação Científica / Conselho Nacional de Desenvolvimento Científico e Tecnológico (CNPq).

\section{REFERÊNCIAS}

1. Uijtendaal E, Van Harssel LLM, Hugenholtz GWK, Kuck EM, Zwart-Van Rijkom JEF, Cremer OL, et al. Analysis of potential drug-drug interactions in medical intensive care unit patients. Pharmacotherapy. 2014;34(3):213-9.

2. Hennessy S, Leonard C, Gagne JJ, Flory JH, Han $X$, Brensinger $C M$, et al. Pharmacoepidemiologic Methods for Studying the Health Effects of Drug-Drug Interactions. Clin Pharmacol Ther. 2016;99(1):92-100.

3. Guthrie B, Makubate B, Hernandez-Santiago V, Dreishculte T. The rising tide of polypharmacy and drug-drug interactions: population database analysis 19952010. BMC Med. 2015;13:74. 
4. Askari M, Eslami S, Louws M, Wierenga PC, Dongelmans DA, Kuiper RA, et al. Frequency and nature of drug-drug interactions in the intensive care unit. Pharmacoepidemiol Drug Saf. 2013;22(4):430-7.

5. Secoli SR, Figueras A, Lebrao ML, de Lima FD, Santos JLF. Risk of potential drug-drug interactions among Brazilian elderly: a population-based, cross-sectional study. Drugs Aging. 2010;27(9):759-70.

6. Fitzmaurice MG, Wong A, Akerberg H, Avramovska S, Smithburger PL, Buckley MS, et al. Evaluation of Potential Drug-Drug Interactions in Adults in the Intensive Care Unit: A Systematic Review and Meta-Analysis. Drug Saf. 2019;42(9):1035-44.

7. Cortes ALB, Silvino ZR, Santos FBM, Pereira JAC, Tavares GS. Drug Interactions Prevalence Involving High-Surveillance Drugs: a Cross-Sectional Study. Rev Min Enferm. 2019;23:e-1226.

8. Reis AMM, Cassiani SH de B. Prevalence of potential drug interactions in patients in an intensive care unit of a university hospital in Brazil. Clinics. 2011;66(1):9-15.

9. Carvalho REFL, Reis AMM, Faria LMP, Azevedo Zago $\mathrm{KS}$, Cassiani SHB. Prevalence of drug interactions in intensive care units in Brazil. Acta Paul Enferm. $2013 ; 26(2): 150-7$.

10. Pessoa T de L, Clemente Junior WS, Costa TX da, Bezerra PK do V, Martins RR. Drug interactions in maternal intensive care: prevalence, risk factors, and potential risk medications. Einstein (Sao Paulo). 2019;17(3):1-7.

11. Janković SM, Pejčić A V., Milosavljević MN, Opančina VD, Pešić N V., Nedeljković $T$, et al. Risk factors for potential drug-drug interactions in intensive care unit patients. J Crit Care. 2018;43:1-6.

12. Rodrigues AT, Stahlschmidt R, Granja S, Pilger D, Falcão ALE, Mazzola PG. Prevalence of potential drug-drug interactions in the intensive care unit of a Brazilian teaching hospital. Brazilian J Pharm Sci. 2017;53(1):e16109.

13. Iyer S, Wagh BR, Godbole DD, Deshmukh SS, Deshpande PR. Identification and Assessment of Potential DrugDrug Interactions in Intensive Care Unit Patients. Indian J Crit Care Med. 2019;23(4):170-4.

14. Rodrigues AT, Stahlschmidt R, Granja $S$, Falcão ALE, Moriel P, Mazzola PG. Clinical relevancy and risks of potential drug-drug interactions in intensive therapy. Saudi Pharm J. 2015;23(4):366-70.

15. Łój P, Olender A, Ślęzak W, Krzych ŁJ. Pharmacokinetic drug-drug interactions in the intensive care unit - single-centre experience and literature review. Anaesthesiol Intensive Ther. 2017;49(4):259-67.

16. Shakeel F, Khan JA, Aamir M. Relationship of factors affecting clinically important drug interactions and their significance in surgical intensive care units in Pakistan. Lat Am J Pharm. 2018;37(4):643-50.
17. von Elm E, Altman DG, Egger M, Pocock SJ, Gøtzsche PC, Vandenbroucke JP. The strengthening the reporting of observational studies in epidemiology (STROBE) statement: Guidelines for reporting observational studies. Int J Surg. 2014;12(12):1495-9.

18. Micromedex $®$ Healthcare Series. Greenwood Village (US): Thomson Reuters (Healthcare) [Internet]. [cited 2019 Nov 5]. Available from: https://www-micromedexsolutions-com.ez32.periodicos.capes.gov.br

19. World Health Organization. Collaborating Center for Drug Statistics Methodology. ATC/DDD Index 2019 [Internet]. [cited 2019 Jan 24]. Available from: https://www.whocc.no/atc_ddd_index/

20. Farzanegan B, Alehashem M, Bastani M, Baniasadi S. Potential drug-drug interactions in cardiothoracic intensive care unit of a pulmonary teaching hospital. J Clin Pharmacol. 2015;55(2):132-6.

21. Farrokh S, Tahsili-Fahadan P, Ritzl EK, Lewin JJ, Mirski MA. Antiepileptic drugs in critically ill patients. Crit Care. 2018;22(1):153.

22. Wang $H$, Wang $C$, Wang $Y$, Tong $H$, Feng $Y$, Li $M$, et al. Sedative drugs used for mechanically ventilated patients in intensive care units: a systematic review and network meta-analysis. Curr Med Res Opin. 2019;35(3):435-46.

23. Panahi Y, Dehcheshmeh HS, Mojtahedzadeh M, JoneidiJafari N, Johnston TP, Sahebkar A. Analgesic and sedative agents used in the intensive care unit: A review. J Cell Biochem. 2018;119(11):8684-93.

24. Instituto para Práticas Seguras no Uso de Medicamentos. Medicamentos potencialmente perigosos de uso hospitalar - lista atualizada 2019. Vol. 8, Boletim ISMP Brasil. 2019. p. 1-9.

25. Reis MAS, Gabriel CS, Zanetti ACB, Bernardes A, Laus AM, Pereira LRL. Potentially hazardous drugs: Identification of risks and error prevention barriers in intensive care. Texto e Context Enferm. 2018;27(2):e5710016.

26. Reichheld AM, Hills-Evans K, Sheehan JK, Tocci NX, Tandon M, Hsu D, et al. A national survey of approaches to manage the ICU patient with opioid use disorder. J Crit Care. 2019;54:42-7.

27. Andrade S, Bartels DB, Lange R, Sandford L, Gurwitz J. Safety of metamizole: a systematic review of the literature. J Clin Pharm Ther. 2016;41(5):459-77.

28. Kötter $T$, Da Costa BR, Fässler M, Blozik E, Linde K, Jüni $P$, et al. Metamizole-associated adverse events: A systematic review and meta-analysis. PLoS One. 2015;10(4):e0122918.

29. Scrignoli CP, Teixeira VCMC, Leal DCP. Drug interactions among the most prescribed drugs in adult intensive care unit. Brazilian J Hosp Pharm Heal Serv. $2016 ; 7(2): 26-30$. 
30. Currow DC, Vella-Brincat J, Fazekas B, Clark K, Doogue M, Rowett D. Pharmacovigilance in hospice/palliative care: Rapid report of net clinical effect of metoclopramide. J Palliat Med. 2012;15(10):1071-5.

31. Simpson PM, Bendall JC, Middleton PM. Review article: Prophylactic metoclopramide for patients receiving intravenous morphine in the emergency setting: A systematic review and meta-analysis of randomized controlled trials. Emerg Med Australas. 2011;23(4):452-7.

32. Marquet K, Claes N, De Troy E, Kox G, Droogmans M, Vleugels A. A multicenter record review of in-hospital adverse drug events requiring a higher level of care. Acta Clin Belgica Int J Clin Lab Med. 2017;72(3):156-62.

33. Phansalkar S, Desai A, Choksi A, Yoshida E, Doole J, Czochanski $M$, et al. Criteria for assessing high-priority drug-drug interactions for clinical decision support in electronic health records. BMC Med Inform Decis Mak. 2013;13:65

34. Paterno MD, Maviglia SM, Gorman PN, Seger DL, Yoshida E, Seger AC, et al. Tiering Drug-Drug Interaction Alerts by Severity Increases Compliance Rates. J Am Med Informatics Assoc. 2009;16:40-6. 